\title{
Typen von Weiterbildungseinrichtungen - Überblick und Ausdifferenzierungen für die Programm- und Organisationsforschung
}

\author{
Aiga von Hippel • Maria Stimm
}

Eingegangen: 17. Februar 2020 / Angenommen: 23. Juni 2020 / Online publiziert: 17. Juli 2020

(C) Der/die Autor(en) 2020, korrigierte Publikation 2020

Zusammenfassung Ziel des Beitrags ist es, ein Angebot zu formulieren, wie Weiterbildungseinrichtungen unter den Entwicklungen der Weiterbildungslandschaft typisiert und systematisch weiter ausdifferenziert werden können. In den Ausführungen wird neben einem Vorschlag zur Ausdifferenzierung eine Erweiterung der bisher bestehenden Perspektive von Einrichtungstypologien angeboten, indem beigeordnete Bildung als Kategorie der Typenbildung mit aufgenommen wird. Der Beitrag verortet sich daher an der Schnittstelle von Programm- und Organisationsforschung.

Schlüsselwörter Programmforschung · Organisationsforschung ·

Einrichtungstypen · Partikularer Typ · Beigeordnete Bildung

Prof. Dr. A. von Hippel · Dr. M. Stimm $(\bowtie)$

Humboldt-Universität zu Berlin, Berlin, Deutschland

E-Mail: maria.stimm@hu-berlin.de

Prof. Dr. A. von Hippel

E-Mail: aiga.von.hippel@hu-berlin.de 


\title{
Types of institutions of further education-A survey of existing types and possibilities of differentiation for use in program research and organizational research
}

\begin{abstract}
The contribution aims at outlining a proposition as to how institutions of further education may be typed and systematically increasingly differentiated within the framework of developments on the landscape of further education. In addition to explaining how this differentiation could be achieved, the article also offers a broadening of the current perspective of typologies of institutions by including adjunctive education as a category of typification. The approach is thus anchored at the interface between program research and organizational research.
\end{abstract}

Keywords Program research - Organizational research - Typology of institutions · Particular type $\cdot$ Adjunctive education

\section{Einleitung}

Programmarchive und -sammlungen der Erwachsenenbildung ${ }^{1}$ stellen vor allem für die Programm- und Organisationsforschung Weiterbildungsprogramme als elementare (historische) Forschungsprimärdaten zur Verfügung. Derzeit gibt es jedoch nur drei Programmarchive und -sammlungen: das Weiterbildungsprogramm-Archiv Berlin/Brandenburg an der Humboldt-Universität zu Berlin, das Programmarchiv des Deutschen Instituts für Erwachsenenbildung - Leibniz-Zentrum für Lebenslanges Lernen und das Österreichische Volkshochschularchiv (s. auch Helmig und Freide 2019).

Das Weiterbildungsprogramm-Archiv Berlin/Brandenburg ist die einzige Sammlung im deutschsprachigen Raum, die Weiterbildungsprogramme von unterschiedlichen Einrichtungstypen archiviert (vgl. Gieseke et al. 2018). Genau an diesem Punkt setzt der Beitrag an und geht der umfassenden Frage nach, welche Typen von Weiterbildungseinrichtungen sich erschließen lassen, um die aktuelle Weiterbildungslandschaft sowie ihre Dynamik darstellen zu können. Zur Untersuchung dieser Fragestellung werden organisationsbezogene Kategorien angelegt.

Folgende Fragestellungen werden in den Ausführungen näher erläutert:

- Welche Typen von Weiterbildungseinrichtungen lassen sich bisher anhand unterschiedlicher Kategorien beschreiben? (Rekurs auf den Theorie- und Forschungsstand)

- Wie könnte die bisherige Unterscheidung in öffentliche, partikulare und private Typen von Weiterbildungseinrichtungen systematisch weiter ausdifferenziert werden? Welche Differenzierungen und weiteren Perspektiven sollten mit aufgenommen werden?

\footnotetext{
1 Wir verstehen „Erwachsenenbildung“ als umfassenden Begriff und nutzen den Begriff „Weiterbildung“ im Sinne eines Systembegriffs u. a. in Komposita.
} 
Ziel des Beitrags ist es damit, ein Angebot zu formulieren, wie unterschiedliche Einrichtungstypen ${ }^{2}$ unter den aktuellen Entwicklungen der Weiterbildungslandschaft gefasst werden können, um anschlussfähig für zukünftige Studien in der Programmund Organisationsforschung zu sein.

In der Programm(planungs)forschung werden das Planungshandeln und das Ergebnis dieses Handelns in Form von Programmen analysiert. Es geht um Gestaltungsräume, steuernde Einflussnahmen und inhaltliche Ausrichtungen. In der Organisationsforschung zeigen Systematisierungsbeiträge auf, dass ,vor allem Formen des Managements und/oder typische Handlungsweisen im Inneren der Organisation, die Beziehung der Weiterbildungsorganisation zu ihrer Umwelt sowie Veränderungen von Weiterbildungsorganisationen" (Herbrechter und Schrader 2018, S. 301) analytisch in den Blick geraten. Die Fokussierung der Entwicklung von Einrichtungen vor historischem, sozialem und/oder politischem Hintergrund bildet dann eine Schnittmenge dieser beiden erwachsenenpädagogischen Forschungsstränge, in der sich auch der folgende Beitrag bewegt.

Die Typisierung von Einrichtungen folgt dabei keinem Selbstzweck, sondern kann helfen, einen (auch retrospektiven und prospektiven) Überblick über die Weiterbildungslandschaft zu gewinnen. Dabei ist die pluralistische Einrichtungsstruktur in der Erwachsenenbildung historisch bedingt und kann mit dem Theorem der ,,mittleren Systematisierung" (Faulstich et al. 1991) beschrieben werden. Es verweist auf die Entwicklungsoffenheit der Weiterbildungslandschaft, aber auch auf deren fehlende Struktur und eine ausstehende politische Stärkung von Erwachsenenbildung als Bildungsbereich.

Daher werden einrichtungsspezifische Typisierungszugänge befragt, die häufig den Dreiklang öffentlich, partikular und privat nutzen (u. a. Wittpoth 2013; Faulstich und Zeuner 1999, 2008) (Abschn. 2). Erkenntnisse aus einer Fragebogenerhebung (Abschn. 3) unterstützen eine theoretisch-empirische Typenbildung. Erste daraus resultierende Ergebnisse und Überlegungen werden vorgestellt (Abschn. 4) sowie einige Systematisierungen (Abschn. 5) erarbeitet.

\section{Bestehende Kategorien für Einrichtungstypologien}

Grundsätzlich lassen sich für die Programm- und Organisationsforschung folgende Kategorien zur Systematisierung unterscheiden: (1) Kategorien zur Strukturierung von Einrichtungen und (2) Kategorien zur Beschreibung von Programmen und Angeboten. Diese zweite Systematisierung findet sich insbesondere in Kodierleitfäden/ Codesystemen von Programmanalysen (s. auch Fleige et al. 2018; von Hippel 2019). Beide Kategorienbereiche sind dabei sowohl für die Programm- als auch für die Organisationsforschung relevant, da sich z.B. über Programmanalysen Einrichtungs-

\footnotetext{
2 Wir sprechen von „Einrichtungstypen“, weil wir in der Untersuchung „Träger“ und „Einrichtungen“ unterschieden haben. Es wäre mit unterschiedlichen Begründungen möglich, von „Organisationstypen“ oder auch „Institutionentypen“ zu sprechen (s. auch Schrader 2011; Tippelt und Lindemann 2018; Herbrechter und Schrader 2018; Faulstich und Zeuner 2008).
} 
profile rekonstruieren lassen; damit wird auch sichtbar, wie in den Einrichtungen Bildung ausgelegt wird.

Wir fokussieren die Kategorien zur Strukturierung von Einrichtungen, die als Typologie angelegt werden können. Eine Typologie ist dabei ,das Ergebnis eines Gruppierungsprozesses, bei dem ein Objektbereich anhand eines oder mehrerer Merkmale in Gruppen bzw. Typen eingeteilt wird“ (Kelle und Kluge 2010, S. 85). Im Gruppierungsprozess wird die Intention verfolgt, Typisches, Ähnlichkeiten, aber auch Unterschiede herauszufiltern, die dann für die Typologie dienen. Grundlegend ist somit die Strukturierung und das Aufdecken von Sinnzusammenhängen der Merkmalskombinationen von Kategorien, aus denen mehrdimensionale Typologien entstehen (Kelle und Kluge 2010, S. 84-85). Die in vermeintlich eindimensionalen Typologien teilweise nicht explizierten Kategorien und Merkmalsräume können auch ex post rekonstruiert werden (s. z. B. zu Handlungsmodi von Programmplanenden Gieseke 1989; von Hippel 2012). Auf den ersten Blick eindimensionale Typologien werden dann in ihrer Mehrdimensionalität deutlich.

Einrichtungstypologien entfalten Relevanz für die Organisationsforschung, aber auch für die Programmforschung. ${ }^{3}$ Gleichzeitig werden sie für Statistiken sowie als Metadaten in Weiterbildungsprogrammarchiven und -sammlungen genutzt.

Dabei bestehen nebeneinander bisher heterogene Systematisierungen nach den folgenden Kategorien, die auch sichtbar machen, dass sich organisationale Strukturen an ganz unterschiedlichen Aspekten ausrichten können: Neben den grundlegenden Systematisierungen in Hauptzweck der Einrichtung, ${ }^{4}$ in Funktionsbereiche (u.a. allgemein, politisch, beruflich), nach inhaltlichen Schwerpunkten (z.B. sprachlich, kulturell, technisch; aber auch monothematisch oder nicht monothematisch) und nach Adressatinnen und Adressaten (vgl. Faulstich und Zeuner 1999, 2008), gibt es ebenso Differenzierungen entlang des Zugangs zur Weiterbildung in offene, also Angebote, die für alle Interessierten zugänglich sind, und geschlossene Angebote ${ }^{5}$ nur für die Belegschaft. Damit verbunden ist auch die rechtliche und gesellschaftliche Stellung des Trägers, indem der geschlossenen Weiterbildungseinrichtung Betriebe, Verbände und öffentlicher Dienst, den offenen Einrichtungen öffentliche (Länder, Kommunen, Hochschulen) sowie nicht-öffentliche Träger (kommerzielle Einrichtungen, freie/gemeinnützige Träger) zugeordnet werden (vgl. Weinberg 1990). Davon zu unterscheiden ist die Rechtsform der Einrichtung. Eine Systematisierung kann neben Einrichtungsgröße, Tätigkeitsbereich (regional oder überregional) und Sitz.

\footnotetext{
3 Ebenso kann der Ausgangspunkt für eine Typisierung aus den Programmen heraus erfolgen. Hier verschieben sich dann die Kategorien für die Typisierung, die über Inhalte, Bildungsverständnis, Programmstruktur u. ä. erfolgt und eine Ableitung für Einrichtungstypen auf dieser Basis ermöglicht.

${ }^{4}$ Der Hauptzweck der Einrichtung kann das Weiterbildungsangebot sein, aber es lassen sich auch andere Hauptzwecke erschließen, die bedingen, dass in diesen Fällen von ,beigeordneter Bildung“ (Fleige et al. 2018), „,nachgeordneter Bildung“ (Wittpoth 2013), ,,impliziter Erwachsenenbildung“ (Kade et al. 2007) oder „sekundärer Erwachsenenbildungsorganisation“ (Schmitz 1980) gesprochen wird.

5 Hier wird eine Schwierigkeit in der Systematisierung deutlich: Beziehen sich die Kategorien auf die gesamte Einrichtung oder auf einzelne Angebote? Als Beispiel dient hier die betriebliche Weiterbildung, die zwar als „geschlossene“ Weiterbildung bezeichnet werden kann, jedoch kommt es häufig vor, dass betriebliche Weiterbildungseinrichtungen ihre freien Plätze einem erweiterten Adressatenkreis anbieten.
} 
der Einrichtung auch die Finanzierungsart und Belegschaftsstruktur in den Blick nehmen.

Eine häufig eingebrachte Systematisierung, in welche Kategorienkombinationen einfließen, ist jene im Spannungsfeld von Staat und Unternehmen. ${ }^{6}$ Daraus ergibt sich eine Differenzierung in öffentliche, partikulare, betriebliche und kommerzielle - die beiden letztgenannten auch zusammengefasst als private - Weiterbildungseinrichtungen als Einrichtungstypen der Weiterbildung (Faulstich und Zeuner 1999, 2008). ${ }^{7}$ Verwiesen werden muss darauf, dass diese Systematisierung teilweise auf der Ebene der Träger und nicht auf der Ebene der einzelnen Einrichtungen angelegt wird. Auch lassen sich zwischen all jenen Typen Überschneidungen finden.

Neben diesen vermeintlich eindimensionalen Systematisierungen liegen aktuell mehrdimensionale, über grundlagentheoretische Einbettung rückgebundene Untersuchungsergebnisse vor. Herbrechter und Schemmann (2010) entwickeln entlang einer Totalerhebung der mittelhessischen Weiterbildungslandschaft Einrichtungstypen auf Basis der Strukturvariablen „Erwerbszweck“, ,,Träger“, „öffentliche Mittel“, „Rechtsform“, „Anbietertyp“, „Gründungsjahr“ und „Themen“. Aus der hierarchischen Clusteranalyse ergeben sich vier übergeordnete Typen von Weiterbildungseinrichtungen: (I) themenvielfältige, öffentliche Anbieter mit langjähriger Bestandsdauer, (II) themenspezifische, private Anbieter mit kurzer Bestandsdauer, (III) Einrichtungen einer Initiative oder eines Vereins mit mittlerer thematischer Orientierung und Bestandsdauer, (IV) Einrichtungen der Wirtschaft mit mittlerer thematischer Orientierung und langjähriger Bestandsdauer (Herbrechter und Schemmann 2010, S. 133-137). In der Interpretation entlang von Temporalstrukturen wird deutlich, „dass zu unterschiedlichen historischen Phasen differente organisationale Felder existieren und zu isomorphen Strukturen geführt haben. Dabei sind die Anpassungen an die Umwelterwartungen derart prägend und nachhaltig, dass die Einrichtungen der jeweiligen Phase noch heute einander ähnlich sind“" (Herbrechter und Schemmann 2010, S. 137).

In der Systematisierung von Schrader (2011) wird eine Typologie in einem Koordinatensystem anhand von Legitimation der Organisation über öffentliche oder private Interessen und Sicherung der Ressourcen der Organisation (durch Vertrag oder Auftrag) vorgelegt. Die dabei jeweils entstehenden Felder dienen als „Reproduktionskontexte der Weiterbildung“ (Schrader 2011, S. 116) dazu, Organisationen einzugruppieren. Grundlegend dafür ist das Anknüpfen an eine neo-institutionalistische Unterscheidung zwischen Institution und Organisation. Aus diesem theoretischen Zugang ergibt sich die „Ebene des institutionellen Umfeldes“ in Schraders „Mehrebenenmodell“ (2011, S. 103). Innerhalb dieser Ebene liegen die aufgeführten Reproduktionskontexte der Weiterbildung.

\footnotetext{
6 Verbunden mit dieser Kategorie von öffentlicher Verantwortung vs. Marktbezug ist die Frage nach den Funktionen und Partialfunktionen, die Einrichtungen für Gesellschaft und Wirtschaft übernehmen (Faulstich und Zeuner 2008).

7 Ähnliche Strukturierungen werden in der Differenzierung öffentliche Träger (Bund, Länder, Kommunen), gesellschaftliche Großgruppen (Kirchen, Parteien, Gewerkschaften, Arbeitgeberverbände, Berufsverbände, Einrichtungen der Wohlfahrtpflege) und private Einrichtungen (z.B. Betriebe, kommerzielle Einrichtungen, Einzelpersonen) angeboten (Gnahs 2001, S. 312).
} 
Insbesondere drei Punkte können jedoch schwer in ihrer Differenziertheit über die aufgezeigten Systematisierungen abgebildet werden: Erstens die beigeordnete Bildung, die nicht in den klassischen Einrichtungen der Erwachsenenbildung zu verorten ist, sondern in Einrichtungen, deren institutioneller Auftrag vordergründig anders gelagert ist. Zweitens - mit der beigeordneten Bildung auch, aber nicht nur zusammenhängend - das rhizomartige Wachstum, welches den ,sich unübersichtlich entwickelnde[n] und entfaltende[n] „Weiterbildungsmarkt" in seiner ungerichteten Offenheit" (Gieseke 2018b, S. 20) beschreibt, auch vor dem Hintergrund des in der Erwachsenenbildung zentralen Subsidiaritätsprinzips. Drittens können hybride Einrichtungen nur schwer bzw. nur über aufwändige Merkmalskombinationen abgebildet werden. Diese drei genannten Punkte dienen als Hintergrundfolie für die Frage des Beitrags nach der Erschließung von Einrichtungstypen in Differenzierung und Erweiterung.

\section{Empirisch fundiertes Vorgehen für eine neue Einrichtungstypologie}

Grundlage des hier folgend kurz vorgestellten Erhebungsprojektes im Rahmen des Weiterbildungsprogramm-Archivs Berlin/Brandenburg ${ }^{8}$ war das Ziel, die Anbieterlandschaft in Berlin und Brandenburg durch eine Einrichtungstypisierung systematisieren zu können, um daran anschließend perspektivische Sammlungsstrategien zu formulieren, die eine inhaltliche Repräsentation der heterogenen Weiterbildungslandschaft im Bestand abdecken. Die Hypothese war, dass sich entlang von Kategorien wie etwa Trägerschaft, Rechtsform, Finanzierung und Zielgruppen empirisch Typen ausbilden lassen. Daher wurden sowohl organisationsbezogene als auch inhaltliche Daten erhoben. Dabei stehen nicht die quantitativen Ergebnisse der Erhebung im Vordergrund (insbesondere da diese nicht statistisch repräsentativ sind), sondern die Ergebnisse dienen uns zur Weiterarbeit an den Typen von Weiterbildungseinrichtungen.

Im Frühjahr 2018 fand ausgehend von der formulierten Zielstellung eine Fragebogenerhebung auf Basis der Einrichtungsdaten des Weiterbildungsprogramm-Archivs Berlin/Brandenburg statt. ${ }^{9}$ Der Fragebogen ist untergliedert in drei Hauptteile: (I) organisationale Angaben, (II) Bildungsangebote und Zielgruppe(n) der Einrichtung sowie (III) allgemeine Angaben zur Einrichtung.

Die Strukturierung des Fragebogens greift einerseits zurück auf eine literaturbasierte Auseinandersetzung mit zentralen Aspekten - u.a. Finanzierungsstrukturen (Hummelsheim 2010; Grotlüschen und Haberzeth 2018; Weiß 2018) und

\footnotetext{
8 Seit 1990 werden rückwirkend im Weiterbildungsprogramm-Archiv Berlin/Brandenburg Programme von mehr als 900 Berliner und Brandenburger Weiterbildungseinrichtungen gesammelt. Diese Sammlung greift damit das gesamte Spektrum der Erwachsenenbildung in seiner Vielfältigkeit für zwei Regionen auf. Demnach bieten sich hier nicht nur Anknüpfungspunkte für die Programmforschung, sondern auch für die Organisations- und Adressatenforschung, die als fall- und/oder themenbezogene Detailanalysen ebenso wie Längsschnittanalysen durchgeführt werden können.

9 Wir danken für die gemeinsame Arbeit daran Iva Jobs, Claudia Kulmus, Tanja Schirmacher und weiteren Mitarbeitenden im Weiterbildungsprogramm-Archiv Berlin/Brandenburg.
} 
Rechtsform ${ }^{10}$ (Wöhe et al. 2016) - sowie auf den Abgleich mit schon bestehenden Antwortmöglichkeiten aus anderen statistischen Erhebungen (von Hippel und Tippelt 2009; Ambos et al. 2013, 2015; Huntemann und Reichart 2017; Martin et al. 2017; Bücker et al. 2017; Horn et al. 2018). Andererseits liegt der Strukturierung des Fragebogens ein zweistufiges Verfahren für die Angaben zum Bildungs- und Veranstaltungsangebot zugrunde. Hier wurden neben den genannten statistischen Erhebungen (ergänzt durch das Berichtssystem Weiterbildung, Bundesministerium für Bildung und Forschung 2006) bestehende, zugängliche Kategoriensysteme aus Programmanalysen (Körber et al. 1995; Gieseke und Opelt 2003; Gieseke et al. 2005; Käpplinger 2007; Schrader 2011) für bestimmte Kategorien gesichtet.

Insgesamt 829 Einrichtungen aus Berlin und Brandenburg, deren Programme zum Zeitpunkt der Erhebung im Weiterbildungsprogramm-Archiv Berlin/Brandenburg gesammelt wurden, wurden für die Fragebogenerhebung angeschrieben. ${ }^{11}$

Auf der Grundlage der insgesamt 225 vorliegenden Fälle ${ }^{12}$ wurde die weitere Datenbearbeitung und -bereinigung vollzogen. ${ }^{13}$ Aus allen Fällen der Online-Umfrage mussten zudem 57 IDs herausgefiltert werden, da teilweise nur Angaben hinsichtlich der ersten und zweiten Frage vorlagen oder Einrichtungen doppelt an der OnlineUmfrage teilgenommen hatten. Somit lagen insgesamt 168 Fälle (bereinigter Stichprobenumfang $N$ ) zur vertieften Analyse vor. Bezogen auf diese 168 Fälle beläuft sich die Rücklaufquote auf $20 \%$.

\section{Aspekte einer neuen Einrichtungstypologie}

Für die Darstellung des Entwurfs einer Einrichtungstypologie, dem eine theoretischempirisch begründete Typenbildung zugrunde liegt, werden wir aufgrund der Fragestellungen einerseits eine Ausdifferenzierung des partikularen Typs (Abschn. 4.1) sowie Überlegungen zu hybriden Einrichtungen (Abschn. 4.2) vorstellen und andererseits eine Erweiterung der bisher bestehenden Perspektive anbieten, indem beigeordnete Bildung als Kategorie der Typenbildung mit aufgenommen wird (Abschn. 4.3). Diese drei Aspekte machen vor dem Hintergrund unterschiedlicher organisationsbezogener Kategorien ebenso Dynamiken innerhalb der Weiterbildungslandschaft sichtbar.

\footnotetext{
10 Wöhe et al. (2016) unterscheiden in der Systematisierung die „Rechtsform privater Betriebe“ (S. 208) und die „Rechtsform öffentlicher Betriebe“ (S. 209). Private Betriebe umfassen Einzelunternehmen, Personalgesellschaften, Kapitalgesellschaften und Genossenschaften, öffentliche Betriebe verweisen auf öffentliche Betriebe in nicht-privatrechtlicher Form (ohne eigene Rechtspersönlichkeit oder mit eigener Rechtspersönlichkeit) sowie öffentliche Betriebe in privatrechtlicher Form (rein öffentliche Betriebe oder gemischtwirtschaftliche Betriebe) (Wöhe et al. 2016, S. 208-209).

${ }_{11}$ Nicht in die Erhebung mit aufgenommen wurden (Solo-)Selbständige, da sie einerseits in der Sammlung nicht repräsentativ abgebildet werden, andererseits keiner Einrichtung angehören.

12 Zwei Fälle wurden aufgrund der fehlenden Repräsentativität und damit einhergehenden Verzerrung aus der Erhebung herausgenommen. Sie waren der betrieblichen Bildung zugeordnet worden.

${ }^{13}$ Hierzu zählen auch die Clusterung der offenen Fragen und des Kommentarfeldes sowie die Nachrecherche von organisationalen Angaben.
} 


\subsection{Partikularer Einrichtungstyp}

Unter dem ersten Hauptteil im vorgestellten Fragebogen greift eine Frage explizit die Erfassung des Einrichtungstyps auf. Hier sollte sich einer erarbeiteten Auswahl an Einrichtungstypen oder einem sonstigen Typ zugeordnet werden. Diese Auswahl von Einrichtungstypen schließt an die Literatur zu Institutionentypen an (s. Abschn. 2, dabei insbesondere Faulstich und Zeuner 1999, 2008), welche die Unterscheidung in öffentlich, partikular, kommerziell sowie betrieblich thematisiert. Mit Blick auf die angestrebte Ausdifferenzierung bestehender Typen von Weiterbildungseinrichtungen nehmen wir in diesem Beitrag nun den partikularen Typ in den Blick. Aus der Feldbeobachtung vermuteten wir, dass der partikulare Typ weiter ausdifferenziert werden könnte, so dass dieser Typ schon im Fragebogen in zwei Untergruppen aufgeteilt wurde.

Zur Auswertung wurden Kreuztabellen (sowie Chi-Quadrat-Tests) genutzt, um einen besseren Überblick über die erhobenen Daten und ihre Zusammenhänge zu gewinnen. ${ }^{14}$ Dabei wurde die Variable zum Einrichtungstyp mit verschiedenen Variablen gekreuzt. Die Chi-Quadrat-Tests waren signifikant bezogen auf „Rechtsform der Einrichtung“ und „Rechtsform des Trägers“, ${ }^{15}$,institutionelle Sockelfinanzierung“, unterschiedliche Finanzierungsquellen sowie die grundsätzliche Ausrichtung des Bildungsangebots (allgemein, beruflich, allgemein und beruflich).

Für den partikularen Typ lässt sich nun in einem ersten Zugang eine Ausdifferenzierung festhalten: Zum einen (a) in Einrichtungen, die den Bildungsauftrag einer gesellschaftlichen Großgruppe vertreten. Hierzu zählen Bildungsakademien von Gewerkschaften, Bildungszentren der Industrie- und Handelskammer, aber auch parteinahe oder politische Stiftungen sowie Einrichtungen der konfessionellen Erwachsenenbildung. Dieser Typ zeichnet sich insgesamt dadurch aus, dass die Auslegung gesellschaftlicher Interessen breiter ist. Zum anderen (b) in Einrichtungen, die den Bildungsauftrag einer kleinen gesellschaftlichen Teilgruppe vertreten. Hier haben sich Interessengemeinschaften in strukturierter Form zusammengeschlossen. Die partikulare Auslegung gesellschaftlicher Interessen und die Transformation in Weiterbildungsangebote im Rahmen der Programmplanung erscheinen demnach im Gegensatz zum partikularen Typ (a) spezifischer, enger.

Der partikulare Typ $(a)$ ist in seiner Rechtsform vor allem durch Vereine, weniger private und öffentliche Betriebe nach Wöhe et al. (2016) gekennzeichnet. Es gibt überwiegend keinen gesonderten Einrichtungsträger. Aufgrund der Datenbasis (Einrichtungen aus zwei Bundesländern mit unterschiedlicher Weiterbildungsgesetzgebung) wird darauf verwiesen, dass entweder öffentliche Mittel oder nicht-öffentliche Gelder als ,institutionelle Sockelfinanzierung“ dienen, vorrangig jedoch keine ,institutionelle Sockelfinanzierung“ vorliegt. Neben den öffentlichen Mitteln, die hier vorrangig Mittel des Bundes oder Mittel des Bundeslandes umfassen, wird auf

\footnotetext{
14 Wir führten auch explorative Clusteranalysen durch, aufgrund der geringen Fallzahl und insbesondere aufgrund der hybriden Einrichtungen verfolgten wir diese Methode allerdings nicht weiter. In einer nachfolgenden, umfangreicheren Erhebung könnten jedoch Clusteranalysen eingesetzt werden.

15 Siehe zu repräsentativen Erhebungen zu Rechtsform in verschiedenen Typen exemplarisch Schrader (2011, S. 117).
} 
Teilnahmegebühren (ohne [Weiter-]Bildungsgutschein) zurückgegriffen. Mitgliedsbeiträge wie Eigenmittel spielen hingegen eine geringe Rolle. Wird Erwachsenenbildung vorrangig als Kerngeschäft beschrieben, ist die grundsätzliche Ausrichtung des Bildungsangebots fast paritätisch aufgeteilt und wird den drei Möglichkeiten allgemein, beruflich sowie allgemein und beruflich zugeordnet.

Der partikular-spezifische Typ (b) ist ähnlich wie Typ (a) in der Rechtsform durch Vereine, weniger durch private Betriebe nach Wöhe et al. (2016) geprägt. Auch hier gibt es keinen gesonderten Einrichtungsträger; wenn doch, dann wird auch dieser Träger in der Rechtsform als Verein beschrieben. Die Abhängigkeit von einem Träger findet sich in Bezug auf die Systematik von Schrader (2011) vor allem im Bereich von Staat (Sicherung von Legitimation durch öffentliches Interesse und der Ressourcen durch einen Auftrag) und Gemeinschaft (Sicherung von Legitimation durch öffentliches Interesse und der Ressourcen durch einen Vertrag), seltener im Kontext von Unternehmen (Sicherung von Legitimation durch privates Interesse und der Ressourcen durch einen Auftrag) und Markt (Sicherung von Legitimation durch privates Interesse und der Ressourcen durch einen Vertrag). Partikular-spezifische Einrichtungen liegen damit an der Schnittstelle von Gemeinschaft und Markt.

Dieser Typ (b) unterscheidet sich von Typ (a) insbesondere darin, dass hier weniger Einrichtungen über eine ,institutionelle Sockelfinanzierung“ verfügen. Wenn beim Typ (b) auf öffentliche Mittel zurückgegriffen wird, dann vorrangig auf Mittel des Bundeslandes. Mitgliedsbeiträge spielen ebenso wie beim partikularen Typ (a) eine Rolle, Eigenmittel jedoch gar nicht. Gegenüber allen anderen Einrichtungstypen sind Teilnahmegebühren für den Typ (b) nicht von Belang. Ebenso wie der partikulare Typ (a) ist die grundsätzliche Ausrichtung des Bildungsangebots fast gleichwertig entlang der drei Möglichkeiten (allgemein, beruflich sowie allgemein und beruflich) aufgegliedert, auch wenn die Tendenz eher zu allgemein oder beruflich geht. Beim Bildungsangebot wird auch hier als Kerngeschäft die Erwachsenenbildung deutlich, aber es gibt stärkere Tendenzen, diese auch als nebengeordnet zu beschreiben.

Werden öffentlich verantwortete, partikulare, partikular-spezifische Typen unter dem Aspekt des öffentlichen Interesses auf einem Kontinuum (auch Schrader 2011) betrachtet, so sind beispielhaft die Volkshochschulen (öffentlich verantworteter Typ) die Einrichtungen mit der breitesten öffentlichen Verantwortung im Sinne kommunaler öffentlicher Daseinsvorsorge und berufen sich dabei auf öffentliche, dem Gemeinwohl verpflichtete Interessen. Diese öffentliche Verantwortung beinhaltet auch eine entsprechende Auslegung von Bildung auf Ebene der Angebote (regionale Verortung, offener Zugang für alle, breites Themenangebot etc.). Danach würde sich der partikulare Typ einsortieren, dessen öffentliche Interessen partikular ausgelegt werden. Der partikular-spezifische Typ legt Bildung (Ziele etc.) spezifischer, enger (meist bezogen auf Zielgruppen und Themen) aus. Dieser Typ nimmt Partialfunktionen wahr und hat dabei eine Funktionalität für spezifische Interessen (Faulstich und Zeuner 2008, S. 185).

Aus der Darstellung dieser Ausdifferenzierung ergeben sich weitere Fragen bezogen auch auf die anderen genannten Typen von Weiterbildungseinrichtungen:

- Welche inneren Einrichtungsstrukturen lassen sich für die Einrichtungstypen nachzeichnen? 
- Gibt es differente Angebots- und Programmstrukturen zwischen den Einrichtungstypen, die wiederum Rückschlüsse auf das Spezifische des Typs zulassen?

Es geht hierbei um die Rekonstruktion von Bildungsinstitutionalkonzepten, Lernkulturen und damit übergreifend um die Auslegung von Bildung, wie sie sich in Programmen zeigt (Fleige et al. 2018, S. 90, S. 111).

\subsection{Hybride Einrichtungen an Schnittstellen der Einrichtungstypen}

Bei der Auswertung und Zuordnung der Einrichtungen zu Typen fallen auch jene Einrichtungen auf, die an der Schnittstelle unterschiedlicher Typen liegen. Sie kennzeichnen, dass sich die Einflussgrößen auf Erwachsenenbildung verändern. Einrichtungen reagieren dynamisch auf Bedarfe, Bedürfnisse und Interessen. Hierzu zählen z. B. die Weiterbildungszentren der Universitäten. Sie liegen - je nach Ausgestaltung und Angebot - an der Schnittstelle von öffentlich verantwortetem Typ (Universität als öffentlicher Betrieb), kommerziellem Typ (kommerzielle Angebote der universitären Weiterbildungszentren) und betrieblichem Typ (Angebote für Belegschaft der Universität). Auch die Übergänge der beiden partikularen Typen sind bei manchen Einrichtungen fließend. Der partikular-spezifische Typ weist insbesondere im Bereich der beruflichen Weiterbildung für spezifische Berufsgruppen (u. a. Verein für die Fortbildung von Erzieherinnen und Erziehern) Schnittstellen mit kommerziellen Weiterbildungseinrichtungen auf.

Deutlich wird an diesen Beispielen, dass die Schnittstellenbereiche der hybriden Einrichtungen spezifiziert werden müssen - entlang inhaltlich-systematischer oder interessenbezogener Differenzierungen. Als weitere Vergleichskategorien könnten daher Zielgruppe oder die Nähe zum formalen Bildungssystem (z. B. Abbilden des Zweiten Bildungswegs, der wissenschaftlichen Weiterbildung) herangezogen werden, um andere Formen von Hybridität systematisch abbilden zu können. Eine weiterführende Auseinandersetzung mit den hybriden Einrichtungen an Schnittstellen fragt somit danach, ob diese, und wenn ja, wie sie in Abgrenzung zu den anderen Typen von Weiterbildungseinrichtungen beschrieben werden können. Lassen sich dabei immer ganze Einrichtungen zuordnen? Hier könnten z. B. die Programmarten (von Hippel und Röbel 2016) einbezogen werden, um die Hybridität deutlich zu machen. So bieten z.B. Volkshochschulen als öffentlich verantworteter Typ auch "Firmenschulungen“ an, die als Programmart eher dem kommerziellen Typ zugeordnet werden würden.

\subsection{Beigeordnete Bildung}

Die folgenden Ausführungen basieren nicht auf den Ergebnissen der Fragebogenerhebung, sondern stellen systematisch-theoretische Überlegungen dar. Sie sind damit ein Vorschritt zur zusammenführenden Systematisierung in Abschn. 5. In diesem Unterkapitel geht es daher zunächst darum, theoretisch die Kriterien zu klären, die für eine Einrichtungstypologie, die auch die beigeordnete Bildung umfassen soll, berücksichtigt werden müssten. 
Daher zunächst eine Definition: „Eine beigeordnete Bildung liegt vor, wenn der Hauptzweck einer Einrichtung nicht Bildung ist, sondern andere gesellschaftliche und/oder fachliche Aufgaben, aber Bildungsangebote unterstützend, ergänzend oder als Marketing angeboten werden, um die zentrale Aufgabe der Einrichtung zu fundieren und sichtbarer zu machen" (Gieseke 2018a, S. 60). Das heißt, dass beigeordnete Bildung in einem deutlichen Zweck-Mittel-Verhältnis zur Unterstützung eines Anliegens steht. Weiterbildung wird in Funktion zu etwas Anderem gesetzt und verweist damit darauf, dass sie selbst als Bereich keine relative Autonomie in der Entscheidung erhält, dieser Bereich daher oft nicht unabhängig agieren kann. Es geht um Qualifizierungsanforderungen von nicht auf Bildung fokussierten Aufgaben (Gieseke 2018a, S. 60). Demnach ist der Ausgangspunkt des Angebots nicht zwingend das Interesse des Individuums, sondern oft seine Funktionsfähigkeit.

Kennzeichen der beigeordneten Bildung (s. grundlegend Gieseke und Opelt 2005) sind daher häufig punktuelle Angebote, die dynamisch auf Anforderungen reagieren, ohne eine festgeschriebene Struktur zu entwickeln, sie sind gewissermaßen angedockt. Ein weiteres Kennzeichen der beigeordneten Bildung ist es, dass zwar Lernen und Bildung in Organisationen stattfindet, ,diese Organisationen aber eben keine eigenen Institutionalisierungsprozesse als Bildungsorganisation durchlaufen haben und Bildung damit Nebenaufgabe ist" (Fleige und Robak 2018, S. 97). Es handelt sich dabei z. B. um betriebliche Bildung, Bildung in Kirchengemeinden oder in Kultureinrichtungen wie Museen, Opernhäusern und Theatern, in Bibliotheken, Planetarien, Vereinen, aber auch in Cafés und Fitnessstudios. ${ }^{16}$

Definitionskriterien für die beigeordnete Bildung sind somit: (1) Hauptzweck der Einrichtung ist nicht (Erwachsenen-)Bildung, Bildung übernimmt jedoch Funktionen für die bessere Erfüllung der Aufgaben der Einrichtung, (2) es handelt sich eher um punktuelle Bildungsangebote, (3) es hat (noch) kein Institutionalisierungsprozess als Weiterbildungseinrichtung stattgefunden. Diese Kriterien könnten auch als Kontinuum angelegt werden.

In unserer Aufarbeitung des Theoriestandes wurde deutlich, dass diese Definitionskriterien bisher nicht zur weitergehenden Systematisierung der beigeordneten Bildung angewandt wurden, sie aber gleichzeitig für bestimmte Einrichtungen eine Relevanz haben.

\section{Systematisierung: Fragen und Anschlussperspektiven}

Einen möglichen Zugang zu den Typen von Weiterbildungseinrichtungen bietet nun die in Abb. 1 dargestellte Systematisierung. Sie greift alle in der Fragebogenerhebung erfassten Einrichtungstypen auf und differenziert sie auf einer vertikalen Achse entlang von Beispielen aus. Diese vertikale Achse wiederum basiert auf der Verortung des Bildungsbereichs in der Einrichtung: Wird Erwachsenenbildung als Hauptzweck beschrieben oder als beigeordnete Bildung? Denn prinzipiell lässt sich

16 Vergleiche zu netzwerkartig organisierten Einrichtungen der Soziokultur mit integriertem Bildungskonzept Enoch (2020). Diese Einrichtungen sind laut Enoch (2020) zwischen Weiterbildungsorganisationen und Organisationen mit beigeordneter Bildung zu verorten. 


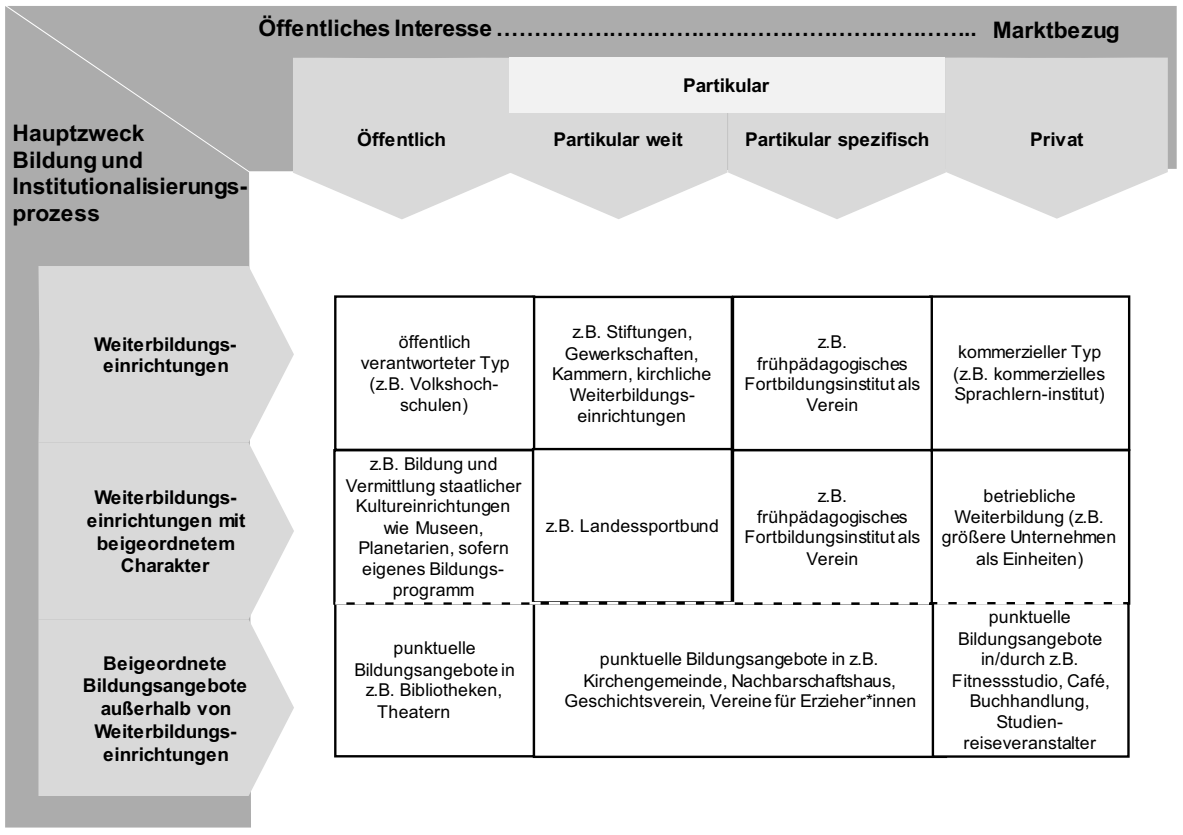

Abb. 1 Typen von Weiterbildungseinrichtungen und Anbietern beigeordneter Bildung

die Unterteilung in öffentlich, partikular und privat auch auf Anbieter beigeordneter Bildung übertragen (s. Abb. 1). Die beigeordnete Bildung lässt sich dafür anhand der festgehaltenen Definitionskriterien (Abschn. 4.3) ausdifferenzieren in Weiterbildungseinrichtungen mit beigeordnetem Charakter und beigeordnete Bildungsangebote außerhalb von Weiterbildungseinrichtungen.

Abb. 1 verdeutlicht nun auch, dass die beiden Vergleichskategorien (Hauptzweck und öffentliches Interesse - Marktbezug) als Kontinuum zu denken sind. Vertikal ist daher eine Trennlinie gestrichelt markiert, da sich hier Entwicklungsprozesse vollziehen können. Solche ,Wanderungsbewegungen“ vertikal, aber auch horizontal, lassen sich über Programmanalysen und statistische Erhebungen nachzeichnen.

Entwickeln Herbrechter und Schemmann (2010) über die durchgeführte Clusteranalyse eine empirische Rückbindung ihrer vier Organisationstypen, bietet sich durch die Typentabelle (s. Abb. 1) eine logisch-systematische Darstellung.

Deutlich wird in der Verschränkung der in Abb. 1 dargestellten Typen von Weiterbildungseinrichtungen und der beigeordneten Bildung, dass Einrichtungen in ihrer Spezifizierung sowohl als Weiterbildungseinrichtung mit beigeordnetem Charakter als auch als Anbieter beigeordneter Bildung einsortiert werden können, je nach vorhandenem Programm und Institutionalisierungsprozess. Das heißt auch, dass sich Anbieter beigeordneter Bildung hin zu Weiterbildungseinrichtungen mit beigeordnetem Charakter institutionalisieren könnten. Weitere Programmforschung macht dann sichtbar, welche Benennung für die Ausdifferenzierung der beigeordneten Bildung tragfähig ist und welche Spezifizierungen sich ergeben. Programmforschung bietet dabei insgesamt den Zugang zu den Programmstrukturen der Einrichtungstypen. 
Dann kann die Fragen verfolgt werden, welche Entwicklungen sich mit welchem Bildungsverständnis und welchen Finanzierungen sowie Institutionalisierungen zeigen.

Die vorgelegte Typologie bietet für Forschungszugänge damit eine Ausdifferenzierung der Einrichtungen an, hier insbesondere jener Einrichtungen, die dem partikularen Typ zugeordnet werden. Weiterführend könnte danach gefragt werden, inwiefern die Typen homogen sind und wo es Heterogenität innerhalb eines Typs gibt. Gleichzeitig ermöglicht die Typologie durch den Einbezug der beigeordneten Bildung einen Gesamtblick auf die Weiterbildungslandschaft.

Deutlich wird in der differenzierten Darstellung der Typen von Weiterbildungseinrichtungen, dass jeder Typ in seiner Mehrdimensionalität wahrgenommen werden muss und sich auf dieser Basis Differenzierungen innerhalb der Typen ergeben. Demnach kann zwar an bisherige Ausdifferenzierungen von Typen von Weiterbildungseinrichtungen angeschlossen werden, aber diese werden nun spezifischer, kleinteiliger ausgestaltet. Genau diese spezifische Ausgestaltung verweist gleichzeitig auf die Strukturen der Weiterbildungslandschaft in ihrem rhizomartigen Wachstum. Es bedarf weiterer Forschungszugänge, um das spezifisch Strukturelle der Erwachsenenbildung nicht nur sichtbar zu machen, sondern ihr dadurch auch den entsprechenden Raum zu geben, den sie eigentlich schon einnimmt.

Open Access Dieser Artikel wird unter der Creative Commons Namensnennung 4.0 International Lizenz veröffentlicht, welche die Nutzung, Vervielfältigung, Bearbeitung, Verbreitung und Wiedergabe in jeglichem Medium und Format erlaubt, sofern Sie den/die ursprünglichen Autor(en) und die Quelle ordnungsgemäß nennen, einen Link zur Creative Commons Lizenz beifügen und angeben, ob Änderungen vorgenommen wurden.

Die in diesem Artikel enthaltenen Bilder und sonstiges Drittmaterial unterliegen ebenfalls der genannten Creative Commons Lizenz, sofern sich aus der Abbildungslegende nichts anderes ergibt. Sofern das betreffende Material nicht unter der genannten Creative Commons Lizenz steht und die betreffende Handlung nicht nach gesetzlichen Vorschriften erlaubt ist, ist für die oben aufgeführten Weiterverwendungen des Materials die Einwilligung des jeweiligen Rechteinhabers einzuholen.

Weitere Details zur Lizenz entnehmen Sie bitte der Lizenzinformation auf http://creativecommons.org/ licenses/by/4.0/deed.de.

\section{Literatur}

Ambos, I., Koscheck, S., \& Martin, A. (2015). Öffentliche Weiterbildungsförderung von Teilnehmenden. Ergebnisse der wbmonitor Umfrage 2015. Bonn: Bundesinstitut für Berufsbildung. https:// wbmonitor.bibb.de/downloads/Ergebnisse_20160405.pdf. Zugegriffen: 2.7.2020.

Ambos, I., Pehl, K., \& Weiland, M. (2013). Analysen zur Erwachsenenbildung in Sachsen-Anhalt im Auftrag des Kultusministeriums des Landes Sachsen-Anhalt. www.die-bonn.de/doks/2013weiterbildungsgesetz-01.pdf. Zugegriffen: 02.07.2020.

Bücker, N., Fleige, M., \& Seiverth, A. (2017). Evangelische Erwachsenenbildung in der Evangelischen Bildungsberichterstattung. Konzeptionelle Begründungen und empirische Umsetzung unter besonderer Berücksichtigung eines neu entwickelten Kategoriensystems zur Anbietererfassung. Münster/New York: Waxmann.

Bundesministerium für Bildung und Forschung (2006). Berichtssystem Weiterbildung IX. Integrierter Gesamtbericht zur Weiterbildungssituation in Deutschland. Bonn, Berlin: BMBF. https://www.phil-fak. uni-duesseldorf.de/fileadmin/Redaktion/Institute/Sozialwissenschaften/BF/Lehre/SoSe2008/KK/ berichtssystem_weiterbildung_neun.pdf 
Enoch, C. (2020). Das Bildungskonzept netzwerkbasierter Kultureinrichtungen der Erwachsenenbildung. In S. Dietel \& W. Gieseke (Hrsg.), Positionieren in der Erwachsenenbildung/Weiterbildung vor dem Hintergrund einer deutsch-deutschen Biographie. Gedenkband für Dr. sc. Helga Stock (S. 85-94). Hamburg: Kovač.

Faulstich, P., \& Zeuner, C. (2008). Erwachsenenbildung. Eine handlungsorientierte Einführung in Theorie, Didaktik und Adressaten (3. Aufl.). Weinheim und München: Juventa.

Faulstich, P., Teichler, U., Bojanowski, A., \& Döring, O. (1991). Bestand und Perspektiven der Weiterbildung. Das Beispiel Hessen. Weinheim: Deutscher Studien Verlag.

Faulstich, P., \& Zeuner, C. (1999). Erwachsenenbildung. Eine handlungsorientierte Einführung in Theorie, Didaktik und Adressaten. Weinheim und München: Juventa.

Fleige, M., \& Robak, S. (2018). Lernkulturen. In M. Fleige, W. Gieseke, A. von Hippel, B. Käpplinger \& S. Robak, Programm- und Angebotsentwicklung in der Erwachsenen- und Weiterbildung (2. Aufl.). (S. 90-103). Bielefeld: wbv Publikation.

Fleige, M., Gieseke, W., von Hippel, A., Käpplinger, B., \& Robak, S. (2018). Programm- und Angebotsentwicklung in der Erwachsenen- und Weiterbildung (2. Aufl.). Bielefeld: wbv Publikation.

Gieseke, W. (1989). Habitus von Erwachsenenbildnern. Eine qualitative Studie zur beruflichen Sozialisation. Oldenburg: BIS.

Gieseke, W. (2018a). Forschungen zum Programmplanungshandeln. In M. Fleige, W. Gieseke, A. von Hippel, B. Käpplinger \& S. Robak (Hrsg.), Programm- und Angebotsentwicklung in der Erwachsenenund Weiterbildung (2. Aufl. S. 52-63). Bielefeld: wbv Publikation.

Gieseke, W. (2018b). Programm und Angebot. In M. Fleige, W. Gieseke, A. von Hippel, B. Käpplinger \& S. Robak (Hrsg.), Programm- und Angebotsentwicklung (S. 18-27). Bielefeld: W. Bertelsmann.

Gieseke, W., \& Opelt, K. (2003). Erwachsenenbildung in politischen Umbrüchen. Programmforschung Volkshochschule Dresden 1945-1997. Opladen: Leske + Budrich.

Gieseke, W., \& Opelt, K. (2005). Programmanalyse zur kulturellen Bildung in Berlin/Brandenburg. In W. Gieseke, K. Opelt, H. Stock \& I. Börjesson (Hrsg.), Kulturelle Erwachsenenbildung in Deutschland - Exemplarische Analyse Berlin/Brandenburg (S. 43-107). Münster: Waxmann.

Gieseke, W., von Hippel, A., Stimm, M., Georgieva, I., \& Freide, S. (2018). Programmarchive und -sammlungen der Erwachsenenbildung/Weiterbildung. In A. von Hippel \& R. Tippelt (Hrsg.), Handbuch Erwachsenenbildung/Weiterbildung (6. Aufl. S. 451-474). Wiesbaden: Springer VS.

Gieseke, W., Opelt, K., Stock, H., \& Börjesson, I. (2005). Kulturelle Erwachsenenbildung in Deutschland - Exemplarische Analyse Berlin/Brandenburg. Münster: Waxmann.

Gnahs, D. (2001). Träger. In R. Arnold, S. Nolda \& E. Nuissl (Hrsg.), Wörterbuch Erwachsenenpädagogik (S. 312-313). Bad Heilbrunn: Klinkhardt.

Grotlüschen, A., \& Haberzeth, E. (2018). Weiterbildungsrecht. In A. von Hippel \& R. Tippelt (Hrsg.), Handbuch der Erwachsenenbildung/Weiterbildung (6. Aufl. S. 543-563). Wiesbaden: Springer.

Helmig, M., \& Freide, S. (2019). Volkshochschulprogramme als Archivgut: Zugänge und Nachnutzungsmöglichkeiten aus Forschungs- und infrastruktureller Perspektive. Spurensuche, 28, 90-99.

Herbrechter, D., \& Schemmann, M. (2010). Organisationstypen der Weiterbildung. Eine empirische Analyse aus der Perspektive des Neo-Institutionalismus. In K. Dollhausen, T. C. Feld \& W. Seitter (Hrsg.), Erwachsenenpädagogische Organisationsforschung (S. 125-141). Wiesbaden: Springer VS.

Herbrechter, D., \& Schrader, J. (2018). Organisationstheoretische Ansätze in der Erwachsenenbildung. In R. Tippelt \& A. von Hippel (Hrsg.), Handbuch Erwachsenenbildung/Weiterbildung (6. Aufl. S. 295-318). Wiesbaden: Springer VS.

von Hippel, A. (2012). Programmplanung als professionelles Handeln - „Angleichungshandeln“ und „Aneignungsmodi“" im aktuellen Diskurs der Programm- und Professionsforschung. In B. Käpplinger, S. Robak \& S. Schmidt-Lauff (Hrsg.), Engagement für die Erwachsenenbildung - Ethische Bezugnahmen und demokratische Verantwortung. Festschrift für Wiltrud Gieseke (S. 131-143). Wiesbaden: Springer VS.

von Hippel, A. (2019). Programmplanungsforschung: Forschungsüberblick und Skizze zu einem Kompetenzmodell für Planende. Hessische Blätter für Volksbildung, 69(2), 103-121.

von Hippel, A., \& Röbel, T. (2016). Funktionen als akteursabhängige Zuschreibungen in der Programmplanung betrieblicher Weiterbildung. Zeitschrift für Weiterbildungsforschung - Report, 39(1), 1-21. https://doi.org/10.1007/s40955-016-0053-1.

von Hippel, A., \& Tippelt, R. (2009). Fortbildung der Weiterbildner/innen. Eine Analyse der Interessen und Bedarfe aus verschiedenen Perspektiven. Weinheim/Basel: Beltz.

Horn, H., Lux, T., \& Ambos, I. (2018). Weiterbildungsstatistik im Verbund 2016 - Kompakt. Bielefeld: wbv. https://www.die-bonn.de/doks/2018-weiterbildungsstatistik-02.pdf

Hummelsheim, S. (2010). Finanzierung der Weiterbildung in Deutschland. Bielefeld: W. Bertelsmann. 
Huntemann, H., \& Reichart, E. (2017). Arbeitsjahr 2016. Volkshochschul-Statistik, Bd. 55. Bielefeld: W. Bertelsmann.

Kade, J., Nittel, D., \& Seitter, W. (Hrsg.). (2007). Einführung in die Erwachsenenbildung/Weiterbildung (2. Aufl.). Stuttgart: Kohlhammer.

Käpplinger, B. (2007). Abschlüsse und Zertifikate in der Weiterbildung. Bielefeld: W. Bertelsmann.

Kelle, U., \& Kluge, S. (2010). Vom Einzelfall zum Typus. Opladen: Leske + Budrich.

Körber, K., Kuhlenkamp, D., Peters, R., Schlutz, E., Schrader, J., \& Wilckhaus, F. (1995). Das Weiterbildungsangebot im Lande Bremen. Strukturen und Entwicklungen in einer städtischen Region. Untersuchung im Auftrag der Strukturkommission Weiterbildung des Senats der Freien Hansestadt Bremen. Ergebnisse der Kommissionsarbeit. Bd. 3. Bremen: Institut für Erwachsenen-Bildungsforschung Universität Bremen.

Martin, A., Lencer, S., Schrader, J., Koscheck, S., Ohly, H., Dobischat, R., Elias, A., \& Rosendahl, A. (2017). Das Personal in der Weiterbildung. Arbeits- und Beschäftigungsbedingungen, Qualifikationen, Einstellungen zu Arbeit und Beruf. Bielefeld: W. Bertelsmann.

Schmitz, E. (1980). Betriebliche Weiterbildung als Personalpolitik. In A. Weyman (Hrsg.), Handbuch für die Soziologie der Weiterbildung (S. 120-136). Darmstadt: Luchterhand.

Schrader, J. (2011). Struktur und Wandel der Weiterbildung. Bielefeld: W. Bertelsmann.

Tippelt, R., \& Lindemann, B. (2018). Institutionenforschung in der Erwachsenenbildung/Weiterbildung. In I. A. von Hippel \& R. Tippelt (Hrsg.), Handbuch Erwachsenenbildung/Weiterbildung (6. Aufl. S. 521-542). Wiesbaden: Springer VS.

Weinberg, J. (1990). Einführung in das Studium der Erwachsenenbildung (2. Aufl.). Bad Heilbrunn: Klinkhardt.

Weiß, R. (2018). Bildungsökonomie und Finanzierung in der Weiterbildung. In A. von Hippel \& R. Tippelt (Hrsg.), Handbuch der Erwachsenenbildung/Weiterbildung (6. Aufl. S. 565-586). Wiesbaden: Springer.

Wittpoth, J. (2013). Einführung in die Erwachsenenbildung (4. Aufl.). Wiesbaden: Springer.

Wöhe, G., Döring, U., \& Brösel, G. (2016). Einführung in die Allgemeine Betriebswirtschaftslehre (26. Aufl.). München: Vahlen.

Publisher's Note Springer Nature remains neutral with regard to jurisdictional claims in published maps and institutional affiliations. 